\title{
Time evolution of the electron density and temperature in laser-produced plasmas from $\mathrm{YBa}_{2} \mathrm{Cu}_{3} \mathrm{O}_{7}$
}

\author{
S.S. Harilal*, C.V. Bindhu, V.P.N. Nampoori, C.P.G. Vallabhan** \\ Laser Division, International School of Photonics, Cochin University of Science \& Technology, Cochin 682 022, India
}

Received: 23 April 1997/Revised version: 25 August 1997

\begin{abstract}
Laser radiation at $1.06 \mu \mathrm{m}$ from a pulsed Nd:YAG laser was focused onto a multielement $\mathrm{YBa}_{2} \mathrm{Cu}_{3} \mathrm{O}_{7}$ target in vacuum and the plasma thus generated was studied using time-resolved spectroscopic techniques. Line broadening of the $\mathrm{Ba}$ I emission line at $553.5 \mathrm{~nm}$ was monitored as a function of time elapsed after the incidence of a laser pulse on the target. Measured line profiles of barium species were used to infer the electron density and temperature, and the time evolution of these important plasma parameters has been worked out.
\end{abstract}

PACS: $52.50 \mathrm{Jm} ; 52.70 \mathrm{kz}$

Pulsed laser ablation has become a very successful method for the deposition of high-quality superconducting $\mathrm{YBa}_{2} \mathrm{Cu}_{3} \mathrm{O}_{7}$ thin films [1-4]. Because of this and other technological applications, considerable efforts have been made to understand the dynamics of the laser-ablated material. The ablation process is accompanied by formation of a bright plasma plume expanding from the target. The laser-induced plasma is characterized by plasma temperature, density, particle expansion velocities, particle charge states and internal fields due to charge separation during plasma expansion.

Optical methods are the most suitable for performing the deposition process control. However, the solution of the processing control problems depends on understanding the basic physics and chemistry associated with laser-target and particle-particle interaction occurring within the laserinduced plasma plume. The emission spectra obtained from a multicomponent $\mathrm{YBa}_{2} \mathrm{Cu}_{3} \mathrm{O}_{7}$ target show numerous emission lines and bands corresponding to neutral particles, ions and diatomic molecules. The dynamics of the laser-ablated $\mathrm{YBa}_{2} \mathrm{Cu}_{3} \mathrm{O}_{7}$ plume has been studied by various techniques such as optical emission spectroscopy [5-9], streak photography $[10,11]$, optical absorption spectroscopy [12-14],

\footnotetext{
* Present Address: Department of Physics, Sree Narayana College, Punalur 691 305, India

**E-mail: root@cochin.ernet.in
}

optical time-of-flight measurements in the nanosecond timescale range [15-19], interferometry [20], and laser-induced fluorescence [21]. However, most of them have been aimed at identification of ablated species and on the evaluation of their velocity distributions. In our previous experiment it was found that the temporal distributions of various ionic, atomic and molecular species were strongly influenced by the spatial position of the plume from the target and the laser fluence used [15].

Besides the identification of the different species generated, most of the time-resolved measurements have focused on dynamics of ejected species from the surface. In the published studies, however, little attention has been paid to the fundamental parameters of the plasma such as electron temperature $\left(T_{\mathrm{e}}\right)$, electron density $\left(n_{\mathrm{e}}\right)$, and their evolution after the initiation of the laser plasma. Several diagnostic techniques are employed in the determination of $n_{\mathrm{e}}$ and $T_{\mathrm{e}}$, including Thomson scattering [22], emission spectroscopy [2325], Langmuir probe [26, 27], and interferometry [20,28]. In this paper we describe the temporal behaviour of the different aspects of the emission spectra of laser-generated plasma from $\mathrm{YBa}_{2} \mathrm{Cu}_{3} \mathrm{O}_{7}$. Also, the temporal dependence of the two vital parameters, electron temperature and electron density, is deduced. The Stark-broadening method is used for the determination of electron density, and the ratio of line intensities of the successive ionization states is used to elucidate the electron temperature. Using gated-integration techniques we report here the time variation of Stark broadening which directly gives the evolution of the internal electric field within the plasma.

\section{Experimental}

Details of the basic experimental arrangements are given elsewhere $[29,30]$. The plasma was generated by ablation of a high-purity sample of superconducting compound $\mathrm{YBa}_{2} \mathrm{Cu}_{3} \mathrm{O}_{7}$ using pulses of wavelength $1.06 \mu \mathrm{m}$ from a Qswitched Nd:YAG laser with repetition rate $10 \mathrm{~Hz}$ and pulse width $9 \mathrm{~ns}$. The target, in the form of a disc $(20 \mathrm{~mm}$ diameter and $3 \mathrm{~mm}$ thickness), was placed in an evacuated chamber 
provided with optical windows for laser irradiation and spectroscopic observation of the plasma produced from the target. During these studies the pressure inside the vacuum chamber is kept at $\sim 10^{-2}$ pascal. The target was rotated about an axis parallel to the laser beam to avoid nonuniform pitting of the target surface. The bright plasma emission was viewed through a side window at right angles to the plasma expansion direction. A section of the plasma was focused onto the slit of a 1 meter Spex monochromator (model 1704, grating with 1200 grooves per mm blazed at $500 \mathrm{~nm}$, maximum resolution $0.015 \mathrm{~nm}$ ) using appropriate collimating and focusing lenses so as to have one-to-one correspondence with the sampled area of the plasma and the image. The recording was done by using a thermoelectrically cooled Thorn EMI Photo Multiplier Tube (PMT), which was coupled to a "boxcar" averager/gated integrator (Standford Research Systems, SRS 250). The averaged output from the boxcar was fed to a chart recorder, which for the present study averaged out the intensities from 10 pulses. For Stark-broadening studies, the resolution of the monochromator was kept at its maximum by keeping the slit widths at minimum possible values for acceptable signal-to-noise ratios.

\section{Results and discussion}

\subsection{Time-resolved emission spectra}

To understand the detailed aspects of laser-beam interaction with the target material and recombination processes following the laser ablation, time-resolved studies of the emission spectra from the plasma offer the most convenient approach. The noninvasive nature of detection suggests that using the emission as a signal source for process monitoring in laser ablation would be ideally suited for designing an automated laser-ablation, superconducting thin-film deposition apparatus. The well defined highly luminous plasma plume was observed during Nd:YAG laser ablation of $\mathrm{YBa}_{2} \mathrm{Cu}_{3} \mathrm{O}_{7}$ and we note that the plume extended normal to the target surface up to a distance $\sim 3 \mathrm{~cm}$. Time-resolved spectra were recorded by setting the gate width of the boxcar averager to $10 \mathrm{~ns}$ and choosing a section $1 \mathrm{~mm}$ away from the target. Typical spectra, as recorded under different regimes of the plasma expansion, are shown in Figs. 1 and 2. Each spectrum corresponds to the average accumulated data over 10 laser shots and it corresponds to a distance of $1 \mathrm{~mm}$ from the target surface. Prominent emission lines observed, together with corresponding literature values and assignments are also shown.

The laser ablation process can be classified into three regimes: evaporation of the target material, interaction of the evaporated cloud with incident laser beam resulting in cloud heating and plasma formation, and expansion and rapid cooling of the plasma [31]. During the initial stages $(<100 \mathrm{~ns})$, the continuum emission dominates over line emission. As time evolves, the line-to-continuum ratio increases. The continuum radiation, or bremsstrahlung, occurs when a free electron collides with another particle and makes a transition to another free state of low energy, with the emission of a photon. Electron-electron collisions do not produce radiation except at relativistic velocities $[32,33]$. In a plasma which is sufficiently hot, most of the atoms are stripped of all their orbital electrons and hence electron-ion recombination and bremsstrahlung are the dominant emission mechanisms. The situation occurs typically at electron temperatures of a few $\mathrm{eV}$. In general, the main sources of continuum emission are bremsstrahlung radiation and radiative recombination [34].

Beyond $100 \mathrm{~ns}$, the line-to-continuum ratio increases and finally the spectrum consists of mainly ionic and atomic lines. At shorter time delays, ionic species are predominant, but for time delays exceeding $500 \mathrm{~ns}$, molecular species are also observed along with ionic and atomic species. From these studies, it is presumed that the electronically excited states are not produced immediately after laser irradiation. Generally, the densities of the particles in a laser-produced plasma with similar fluence are in the range of $10^{17} \mathrm{~cm}^{-3}$ and these densities are sufficient to justify the assumption of occurrence of collisional processes among the particles in the plasma. As the velocity of the particles in the expanding plume has been found to be high $\left(\sim 5 \times 10^{5} \mathrm{~cm} \mathrm{~s}^{-1}\right.$, which corresponds to $13 \mathrm{eV})[15,35]$, the collision between particles which have high kinetic energy may lead to electronic excitation of the component atoms, ions or molecules. It is also noted that the plasma emission lasts for more than $100 \mu$ s (Figs. 1b and 2b).

Although, at least qualitatively, the results confirm the presence of strong emission lines from Y I, Y II, Ba I, Ba II, $\mathrm{Cu} \mathrm{I}$ and $\mathrm{YO}(\mathrm{A} \rightarrow \mathrm{X})$ in the plasma, features corresponding to $\mathrm{Cu}$ II, $\mathrm{BaO}$ and $\mathrm{CuO}$, may be present, but are very weak. Since these spectra were recorded very close to the target surface $(1 \mathrm{~mm})$, it is understandable that the emission intensities due to oxide species are very weak, since they are expected to be formed only by recombination processes [15]. The relatively weak intensity observed for the $\mathrm{Cu}$ II emission lines is due to the higher ionization potential of a $\mathrm{Cu}$ atom $(7.726 \mathrm{eV})$ compared to $\mathrm{Ba}(5.211 \mathrm{eV})$ and $\mathrm{Y}(6.378 \mathrm{eV})$ [36]. Since the ionization potentials of these lines are much greater than the excitation energy of the laser photons $(1.16 \mathrm{eV})$, the mechanism of ionization is possibly due to a multiphoton process.

\subsection{Electron density measurements}

The usual method for determination of electron density is the measuring of the broadening of a suitable emission line of the laser-plasma spectrum. Stark broadening is the dominant broadening mechanism in laser-produced plasmas. In such a case the electron density can be deduced from the full width at half maximum (FWHM) of a line.

The full width at half maximum, $\Delta \lambda_{1 / 2}$, of a Starkbroadened line without ionic contribution is given by the simple relation [23]

$\Delta \lambda_{1 / 2}=2 W\left(\frac{n_{\mathrm{e}}}{10^{16}}\right) \AA$,

where $W$ is the electron-impact parameter which can be incorporated for different temperatures [23].

We have selected the line-broadened profile of the atomic $\mathrm{Ba}$ at $553.5 \mathrm{~nm}\left(6 \mathrm{p}^{1} \mathrm{P}_{1}^{0}-6 \mathrm{~s}^{1} \mathrm{~S}_{0}\right)$ for the electron-density measurements. There are three types of mechanisms that can broaden an emission line: resonance broadening, Doppler broadening, and Stark broadening. The effect of resonance broadening is proportional to the ground state number density and the transition oscillator strength. Since the reported value for resonance broadening for Ba I at $553.5 \mathrm{~nm}$ is relatively small [37], the contribution due to this type of broadening can 

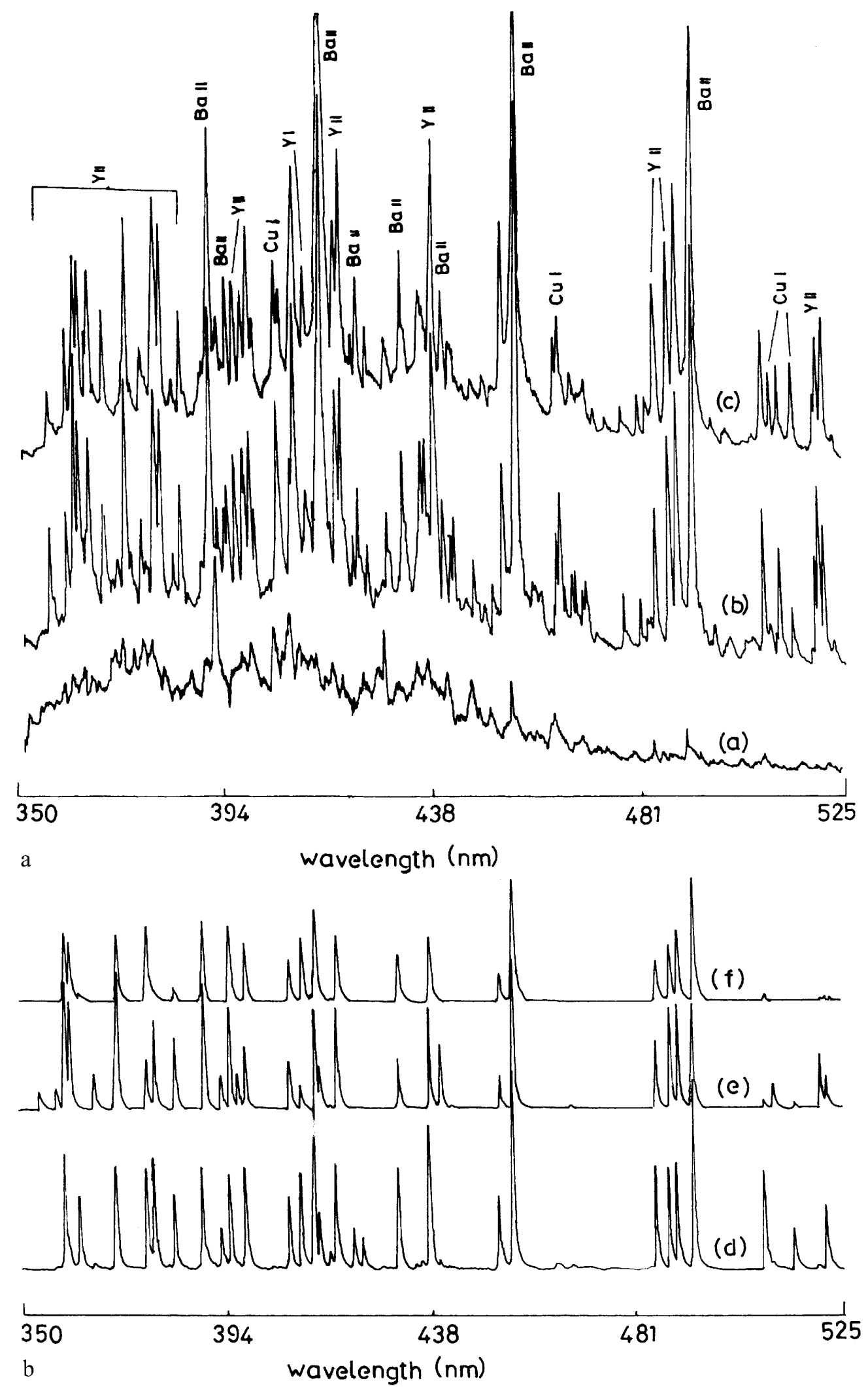

Fig. 1a,b. Plasma emission spectra in the wavelength range $\lambda \lambda$ $350-525 \mathrm{~nm}$ at different time delays (a) $100 \mathrm{~ns}$, (b) $500 \mathrm{~ns}$, (c) $5 \mu \mathrm{s}$, (d) $30 \mu \mathrm{s}$, (e) $50 \mu \mathrm{s}$, and (f) $100 \mu \mathrm{s}$. The spectra are charted at a distance $1 \mathrm{~mm}$ away from the target surface and at a laser fluence of $360 \mathrm{~J} \mathrm{~cm}^{-2}$

be neglected. Doppler broadening, which is due to different Doppler shifts (i.e. $\Delta \lambda=\lambda v_{z} / c$ ) experienced by the species in different regions of the plume having different velocity components $v_{\mathrm{z}}$, can also be ruled out in our experimental conditions, since the expansion velocities of the $\mathrm{Ba}$ atoms are $\sim 5 \times 10^{5} \mathrm{~cm} \mathrm{~s}^{-1}$ [35] which contribute a broadening factor $\sim 0.1 \AA$. Under these conditions Stark broadening, due to collisions with plasma electrons, is the dominant broadening 


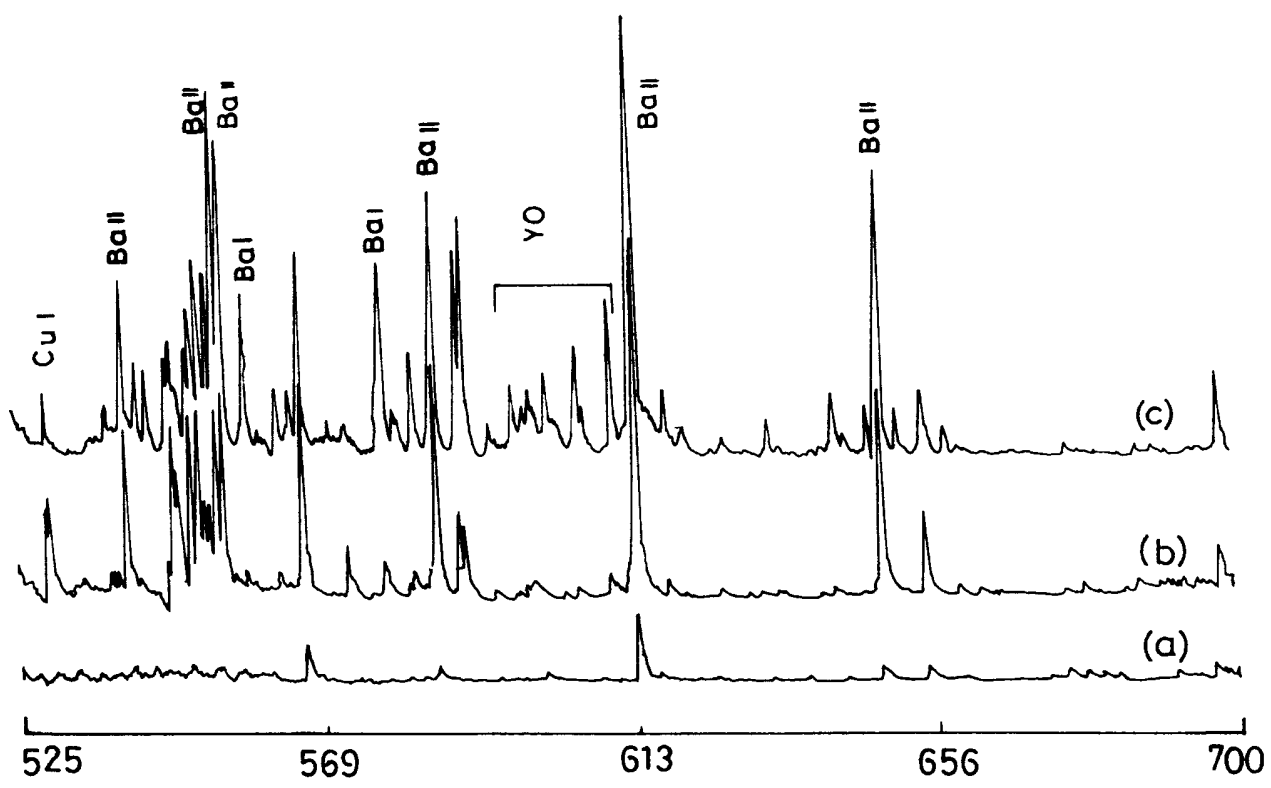

a

Wavelength $(\mathrm{nm})$

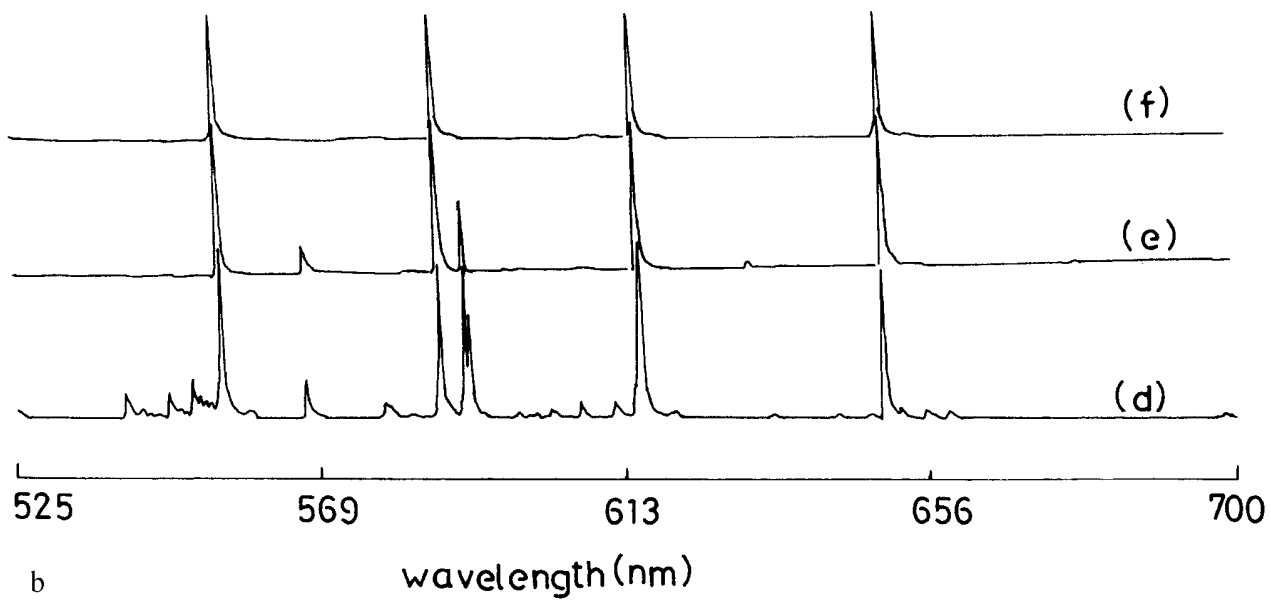

Fig. 2a,b. Time-resolved $\mathrm{YBa}_{2} \mathrm{Cu}_{3} \mathrm{O}_{7}$ plasma emission spectra in the wavelength range $\lambda \lambda 525-700 \mathrm{~nm}$. The spectra are charted at a distance $1 \mathrm{~mm}$ away from the target surface and at a laser fluence of $360 \mathrm{~J} \mathrm{~cm}^{-2}$. Time delays (a) $200 \mathrm{~ns}$, (b) $500 \mathrm{~ns}$, (c) $5 \mu \mathrm{s}$, (d) $30 \mu \mathrm{s}$, (e) $50 \mu \mathrm{s}$ and (f) $100 \mu \mathrm{s}$

process. The electron impact parameter $(W)$ values for the $\mathrm{Ba}$ I $553.5 \mathrm{~nm}$ transition are not available in the literature, but values are available for the corresponding $n s n p^{1} \mathrm{P}_{1}^{0} \rightarrow \mathrm{ns}^{21} \mathrm{~S}_{0}$ transitions of the elements $\mathrm{Mg}$, Be and Ca from the same periodic group [38]. The latter values increase approximately linearly with $n^{2}$ and this dependence was extrapolated to yield a $W$ value of $\sim 1.6 \times 10^{-2} \AA$ for Ba I $553.5 \mathrm{~nm}$ transition at a temperature of $10000 \mathrm{~K}$. Since $W$ values are weak functions of temperature and vary by a factor of less than 2 over the temperature range $10000-80000 \mathrm{~K}$, the determination of electron density deduced using this $W$ value should be reliable.

A typical Stark-broadened profile of Ba I at the $553.5 \mathrm{~nm}$ line with a theoretically fitted Lorentzian curve is given in Fig. 3. We have measured electron density variation using the Stark-broadening method as functions of distance of separation from the target, time after the elapse of the laser pulse, and laser fluence. The variation of electron density as a function of distance $z$ shows a $z^{-1}$ dependence [39] which is in good agreement with the adiabatic expansion model predicted by Singh and Narayan [40]. It is also noted that the values of electron densities are essentially constant at higher laser fluences [39]. The saturation in electron density at higher fluences is presumably due to plasma shielding. The absorbing plasma generated by the leading edge of the laser pulse prevents light from reaching the surface. The prominant mechanism responsible for plasma absorption at these fluence levels is inverse bremsstrahlung [25].

The temporal evolution of electron density deduced from Stark broadening of the Ba I $553.5 \mathrm{~nm}$ transition is shown in Fig. 4. These measurements were made at a distance $3 \mathrm{~mm}$ from the target surface and at a laser fluence of $378 \mathrm{~J} \mathrm{~cm}^{-2}$ (power density $42 \mathrm{GW} \mathrm{cm}^{-2}$ ). For these studies the gate width of the boxcar was set at $10 \mathrm{~ns}$. Because the spectral line emission is masked by the continuum emission, the electron density for time $<200 \mathrm{~ns}$ could not be measured by this method. Nevertheless, we can still characterize the temporal evolution of $n_{\mathrm{e}}$, which diminishes rapidly with time up to $500 \mathrm{~ns}$ and then levels off. The fast decay rate can be attributed to plasma propagation while the slowing and leveling off at larger times 


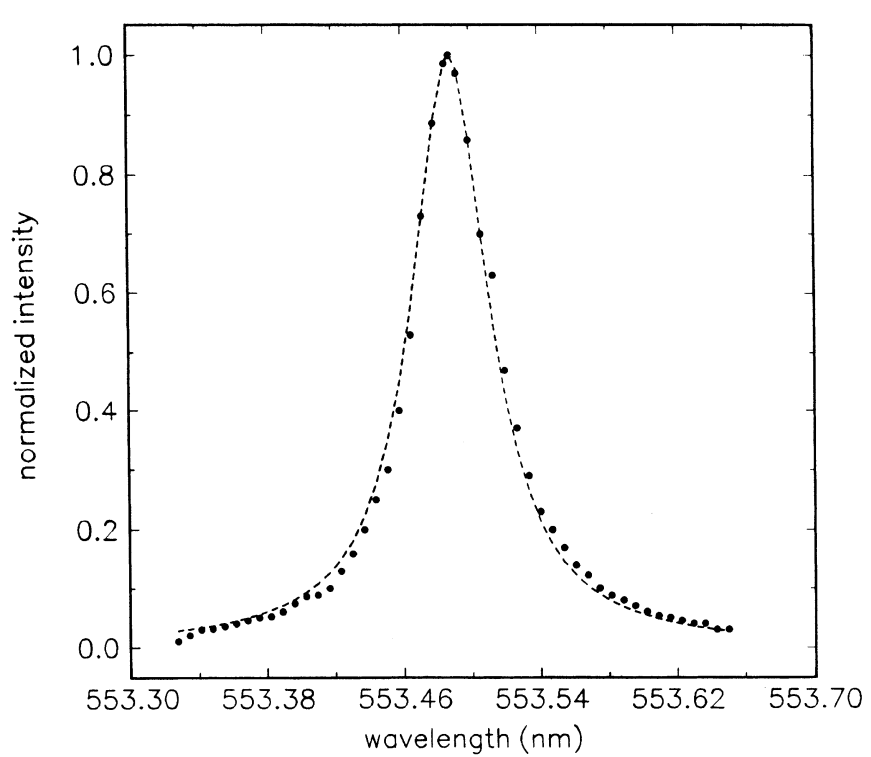

Fig. 3. Typical Stark-broadened profile of Ba I transition at $553.5 \mathrm{~nm}$. The FWHM of this line is used to infer electron density. The dotted line in the figure represents the fitted Lorentzian curve

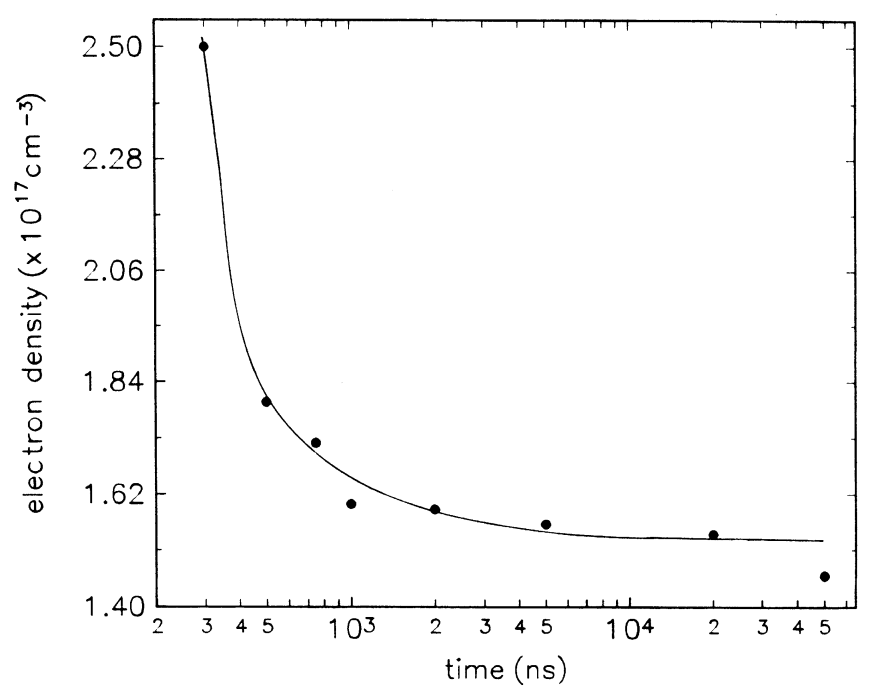

Fig. 4. Time evolution of electron density at $3 \mathrm{~mm}$ from the target surface. Laser fluence used is $378 \mathrm{~J} \mathrm{~cm}^{-2}$

are probably due to recombination. It may also be noted that electron density shows a $t^{-2}$ dependence on time which is in accordance with adiabatic expansion model.

\subsection{Electron temperature measurements}

Electron temperature was determined from the relative intensities of neutral and ionized barium spectral lines. We have used spectral lines of successive ionization states since in this case the energy difference between the upper levels of the lines is increased by the ionization energy of the lower ionization state $[23,26]$. Electron temperature measurements were made using the equation

$\frac{I^{\prime}}{I}=\left[\frac{f^{\prime} g^{\prime} \lambda^{3}}{f g \lambda^{\prime 3}}\right]\left[4 \pi^{3 / 2} a_{0}^{3} n_{\mathrm{e}}\right]^{-1}\left[\frac{k T_{\mathrm{e}}}{E_{\mathrm{H}}}\right]^{3 / 2} \mathrm{e}^{-(\Delta E / k T)}$, where $\Delta E=\left(E^{\prime}-E+E_{\infty}-\Delta E_{\infty}\right)$, the primed quantities represent relevant data for the spectral line emitted by the species with higher ionization state, $E_{\mathrm{H}}$ is the ionization energy of the hydrogen atom $(13.6 \mathrm{eV}), E_{\infty}$ is the ionization energy of the atom or ion in the lower ionization state, and $\Delta E_{\infty}$ is the correction factor of the energy due to plasma interaction which is given by

$\Delta E_{\infty}=3 z \frac{\mathrm{e}^{2}}{4 \pi \varepsilon_{0}}\left[\frac{4 \pi n_{\mathrm{e}}}{3}\right]^{1 / 3}$

where $z=2$ for singly ionized species [23].

For temperature calculations, we make use of recorded intensities for the Ba II $614 \mathrm{~nm}$ and $649.8 \mathrm{~nm}$ lines and the Ba I, $577.7 \mathrm{~nm}$ and $553.5 \mathrm{~nm}$ lines. Details of the spectroscopic constants of these lines were taken from [23]. The electron temperature measurements were carried out as functions of distance from the target surface, time after the initiation of the plasma, and incident laser fluence. The electron temperature follows a $z^{-0.8}$ dependence on distance from the target surface [39]. With increase in laser fluence, the electron temperature is found to increase up to a specific value, beyond which it saturates. The saturation in electron temperature at higher fluences is again due to the absorption of the laser beam by the plasma medium [39].

The electron temperature calculations were made at different instants during the plasma evolution by keeping the gate width of the boxcar averager at $10 \mathrm{~ns}$ but by varying the delays. At shorter time delays, continuum emission occurs and line intensity detection is rather difficult. The continuum stems from the elastic collision of electrons with ions. The intensity of the continuum decreases with increasing delay time. The calculated plasma temperature and its variation as a function of time after the initiation of the plasma are shown in Fig. 5. In the early stage of plasma evolution, the electron temperature is very high and changes very rapidly. However at times $\geq 1 \mu \mathrm{s}$, the plasma is found to be at much lower temperatures and thereafter the temperature continues to remain fairly stable, around $0.6-0.7 \mathrm{eV}$ for a long period. The electron temperature follows a $t^{-2}$ dependence on time dur-

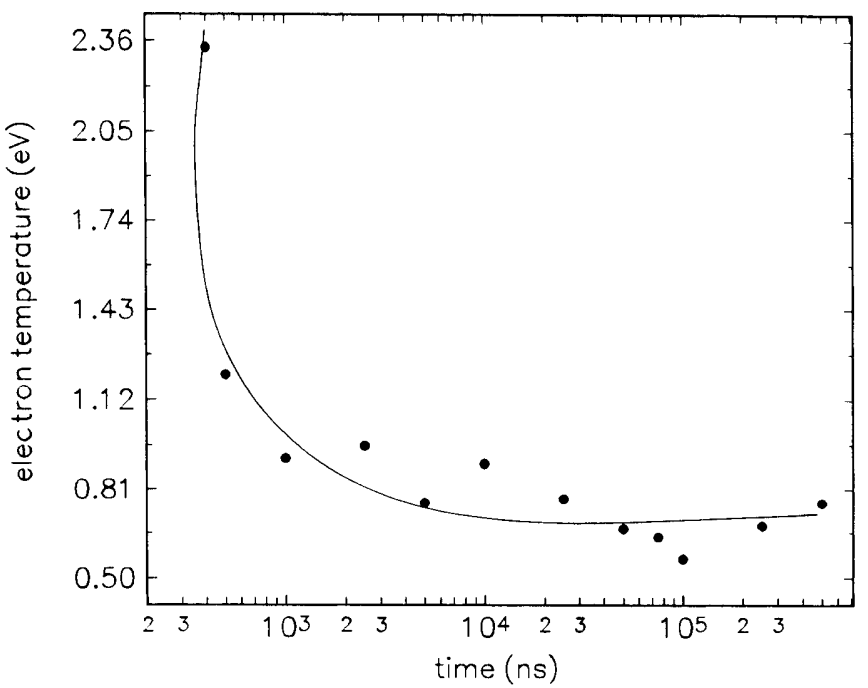

Fig. 5. The variation of electron temperature with time. These spectra were recorded $3 \mathrm{~mm}$ from the target surface and at a laser fluence of $315 \mathrm{~J} \mathrm{~cm}^{-2}$ 
ing the initial periods up to $\sim 1 \mu$ s which is in accordance with the theoretical adiabatic expansion model by Rumbsy and Paul [41].

Since we have assumed that the plasma is in local thermodynamic equilibrium (LTE) for our analysis of electron temperature, it is worthwhile checking the minimum density condition for LTE, [42]:

$n_{\mathrm{e}} \geq 1.4 \times 10^{14} T_{\mathrm{e}}^{1 / 2}(\Delta E)^{3} \mathrm{~cm}^{-3}$

For the transition with the largest gap $(553.5 \mathrm{~nm}), \Delta E=$ $2.24 \mathrm{eV}$ and the highest temperature $2.5 \mathrm{eV}$; the lower limit for $n_{\mathrm{e}}$ is $2.5 \times 10^{15} \mathrm{~cm}^{-3}$. Our observed values of $n_{\mathrm{e}}$ are always greater than this limit implying that the LTE approximation assumed for our analysis is valid.

\section{Conclusions}

The present work has identified the major luminescent species in the plume of $\mathrm{Nd}$ :YAG laser-ablated $\mathrm{YBa}_{2} \mathrm{Cu}_{3} \mathrm{O}_{7}$ as neutral and ionized atoms along with diatomic molecules. We have estimated the electron temperature of the plasma by spectroscopic means. Line intensities of the successive ionization states were used for the determination of the electron temperature and the Stark-broadened profile of the Ba I transition at $553.5 \mathrm{~nm}$ was used for the measurement of electron density. Both the electron temperature and the electron density show a $t^{-2}$ type dependence on the time elapsed after the laser pulse.

Acknowledgements. The present work is partially supported by the Department of Science and Technology, Government of India. SSH and CVB are grateful to the Council of Scientific and Industrial Research, New Delhi and University Grant Commission, New Delhi for financial support.

\section{References}

1. P. Tiwari, S. Sharan, J. Narayan: Appl. Phys. Lett. 59, 357 (1991)

2. D.B. Chrisey, G.K. Hubler (eds.): Pulsed Laser Deposition of Thin Films, (Wiley, New York 1994)

3. R.K. Singh, O.W. Holland, J. Narayan: J. Appl. Phys. 68, 233 (1990)

4. A. Kühle, J.L. Skov, S. Hjorth, I. Rasmussen, J.B. Hansen: Appl. Phys. Lett. 64, 3178 (1994)

5. T.J. Geyer, W.A. Weimer: Appl. Phys. Lett. 54, 469 (1989)

6. P.E. Dyer, R.D. Greenough, A. Issa, P.H. Key: Appl. Phys. Lett. 53, 534 (1989)

7. O. Auciello, S. Athavale, O.E. Hankins, M. Sito, A.F. Schreiner, N. Biunno: Appl. Phys. Lett. 53, 72 (1988)

8. X.D. Wu, B. Dutta, M.S. Hegde, A. Inam, T. Venkatesan, E.W. Chase, C.C. Chang, R. Howard: Appl. Phys. Lett. 54, 179 (1989)
9. W.A. Weimer: Appl. Phys. Lett. 52, 2171 (1988)

10. O. Eryn, K. Murakami, K. Masuda, A. Kasuya, Y. Nichima: Appl. Phys. Lett. 54, 2716 (1989)

11. P.E. Dyer, A. Issa, H. Key: Appl. Phys. Lett. 57, 186 (1990)

12. R.A. Al-Wazzan, J.M. Hendron, T. Morrow: Appl. Surf. Sci. 96-98, 170 (1996)

13. D.B. Geohegan, D.N. Mashburn: Appl. Phys. Lett. 55, 2345 (1989)

14. R.A. Al-Wazzan, J.M. Hendron, T. Morrow: Appl. Surf. Sci. 99, 345 (1996)

15. S.S. Harilal, P. Radhakrishnan, V.P.N. Nampoori, C.P.G. Vallabhan: Appl. Phys. Lett. 64, 3377 (1994)

16. S.S. Harilal, R.C. Issac, C.V. Bindhu, V.P.N. Nampoori, C.P.G. Vallabhan: J. Appl. Phys. 80, 3561 (1996)

17. S.S. Harilal, R.C. Issac, C.V. Bindhu, V.P.N. Nampoori, C.P.G. Vallabhan: J. Appl. Phys. 81, 3637 (1997)

18. S.S. Harilal, R.C. Issac, C.V. Bindhu, V.P.N. Nampoori, C.P.G. Vallabhan: Jpn. J. Appl. Phys. 26, 134 (1997)

19. K.L. Saenger: J. Appl. Phys. 66, 4435 (1989)

20. G.K. Varier, R.C. Issac, C.V. Bindhu, S.S. Harilal, V.P.N. Nampoori, C.P.G. Vallabhan: Spectrochim. Acta, Part B 52, 657 (1997)

21. T. Okada, N. Shibamaru, Y. Nakayama, M. Maeda: Appl. Phys. Lett. 60, 941 (1992)

22. S.B. Cameron, M.D. Tracy, J.P. Camacho: IEEE Trans. Plasma Sci. 24, 45 (1996)

23. H.R. Griem: Plasma Spectroscopy (McGraw-Hill, New York 1964)

24. S.S. Harilal, R.C. Issac, C.V. Bindhu, V.P.N. Nampoori, C.P.G. Vallabhan: J. Phys. D 30, 1703 (1997)

25. S.S. Harilal, R.C. Issac, C.V. Bindhu, V.P.N. Nampoori, C.P.G. Vallabhan: J. Appl. Phys. 82, 2140 (1997)

26. R.H. Huddlestone, S.L. Leonard: Plasma Diagnostic Techniques (Academic, London 1965)

27. J.M. Hendron, C.M.O. Mahony, T. Morrow, W.G. Graham: J. Appl. Phys. 81, 2131 (1997)

28. T. Mochizuki, K. Hirata, H. Ninomiya, K. Nakamura, K. Maeda, S. Horiguchi, Y. Fujiwara: Opt. Commun. 72, 302 (1989)

29. S.S. Harilal, R.C. Issac, C.V. Bindhu, V.P.N. Nampoori, C.P.G. Vallabhan: Pramana - J. Phys. 46, 145 (1996)

30. S.S. Harilal, R.C. Issac, C.V. Bindhu, V.P.N. Nampoori, C.P.G. Vallabhan: Spectrochim. Acta, Part A 53, 1527 (1997)

31. W.K.A. Kumudini, Y. Nakata, T. Okada, M. Maeda: Appl. Phys. B 58, 289 (1994)

32. T.P. Hughes: Plasmas and Laser Light (Adam Hilger, England 1975)

33. L.J. Radziemski, D.A. Cremers: Laser-induced Plasmas and Applications (Marcel Dekker, New York 1989)

34. Y.B. Zeldovich, Y.P. Raizer: Physics of Shock Waves and HighTemperature Hydrodynamic Phenomena (Academic Press, 1966)

35. S.S. Harilal: Ph.D. thesis, Cochin University of Science and Technology (1997)

36. R.C. Weast: CRC Handbook of Chemistry and Physics (CRC Press, Florida 1988)

37. D.B. Geohegan: in Pulsed Laser Deposition of Thin Films, D.B. Chrisey, G.K. Hubler (eds.) (Wiley, New York 1994)

38. A.H. El-Astal, T. Morrow: J. Appl. Phys. 80, 1156 (1996)

39. S.S. Harilal, C.V. Bindhu, V.P.N. Nampoori, C.P.G. Vallabhan: Appl. Spectro., in press (1998)

40. R.K. Singh, J. Narayan: Phys. Rev. B 41, 8843 (1990)

41. P.T. Rumsby, J.W.M. Paul: Plasma Phys. 16247 (1974)

42. G. Bekefi: Principles of Laser Plasmas, (John Wiley \& Sons, New York 1976) 